\title{
Cosine Similarity Measure of Interval Valued Bipolar Neutrosophic Hesitant Fuzzy Set and Their Applications to Multi-Attribute Decision-Making Process
}

\author{
Hans Eric Ramaroson, René Rakotomanana and Hery Zo Andriamanohisoa
}

\begin{abstract}
Cosine similarity measure plays a significant role in various fields. Literature consultation confirms that the theory of cosine similarity measure has received a great interest and attention from the researchers in the world. The concept of Interval Valued Bipolar Neutrosophic Hesitant Fuzzy Sets (IVBNHFS) is recently presented and very interesting. Every element in IVBNHFS is characterized by six independent membership functions (three positive and three negative). There is no investigation on the Cosine Similarity Measure (CSM) of IVBNHFS. In this study, we firstly define a CSM and a weighted CSM between two IVBNHFS and their applications to MultiAttribute Decision Making (MADM) process in the Interval Valued Bipolar Neutrosophic Hesitant Fuzzy (IVBNHF) setting. And, we establish some properties of CSM and a weighted CSM. We use this strategy to find out the best alternative in MADM case. Then, the new approach to clarify MADM problems in IVBNHF setting is presented in algorithmic form. And, we solve an illustrative case of MADM to demonstrate the effectiveness, workability, and appropriateness of the proposed approach. Finally, the main conclusion and future opportunity of research paper are overviewed and compiled.
\end{abstract}

Key words - Cosine Similarity Measure (CSM), weighted CSM, Interval Valued Bipolar Neutrosophic Hesitant Fuzzy Set (IVBNHFS), Similarity measure, Multi-Attribute DecisionMaking (MADM).

\section{INTRODUCTION}

Fuzzy Set (FS) [1] is given officially by Zadeh in 1965. Intuitionistic Fuzzy Set (IFS) [2] is exposed by Atanassov in 1983. Neutrosophic set (NS) [3] is suggested by Smarandache in 1998. It is a generalization of FS [1] and IFS [2]. Zhang proved that Bipolar Fuzzy Sets (BFS) [4] is very efficiency in uncertain problems which can characterized the positive and the negative characteristics. However, Torra introduced Hesitant Fuzzy Set (HFS) [5] in 2010. In 2014, [6] proposed the Hesitant Neutrosophic Sets (HNS) [6] as a generalization of HFS [5]. In 2018, Neutrosophic BFS [7] in daily life's problem is proposed by [7].

About the Cosine Similarity Measure (CSM) subject, many researchers [8]-[19] have worked on this. A CSM between two and weighted Interval Valued Neutrosophic Sets (IVNS) is recommended by [8] in 2014. Also, [8] defined a new Cosine Similarity (CS) between two IVNS based on Bhattacharya's distance. A CSM based MADM with Trapezoidal Fuzzy Neutrosophic Numbers (TFNN) is exhibited by [9]. In 2015, [10] defined a rough CSM between two Rough Neutrosophic Sets (RNS). Later, [11] proposed three cosine measures between Neutrosophic Cubic Sets (NCS). In 2018, [12] introduced a new Analytic Hierarchy Process (AHP) and proposed an Interval Valued Neutrosophic AHP (IVN-AHP) based on CSM. The proposed strategy with CSM by [12] gave a target scoring method to pairwise correlation networks under neutrosophic uncertainty. In the other case, a corresponding Cosine Distance Measure (CDM) between Neutrosophic Hesitant Fuzzy Linguistic Term Sets (NHFLTSs) is proposed by [13] according to the relationship between the similarity measure and the distance measure. Also, [13] developed the Technique for Order Preference by Similarity to an Ideal Solution (TOPSIS) method to the obtained CDM. Whatever, improved CSM for an IFS has been proposed by [14] and [15] proposed a CSM between hybrid IFS. In 2018, [16] proposed Cosine Exponential Distance (CED) among Single Valued Neutrosophic Multi Sets (SVNMS), Cosine Logarithmic Distance (CLD) [17] among single valued NS and some of its properties are discussed. Then, [18] introduced a SingleValued Neutrosophic Multiset (SVNM). Based on the weighted CSM of SVNM [18], a MADM method under a SVNM environment is advanced. In 2019, [19] proposed three types of CSM for resolving MADM problems based on proposed types of CSM [19] with a Bipolar and Interval Bipolar Neutrosophic (BIBN) data. In the field of Hesitant Set (HS), [20] formulate Hesitant Bipolar-Valued Neutrosophic Set (HBVNS). Also, in 2020, [21] firstly introduced the concept of Interval Valued Bipolar Neutrosophic Hesitant Fuzzy Sets (IVBNHFS). However, in 2020 and 2021, Bipolar [22]-[25] and decision making [26][44] has been proposed by many researchers [26]-[44] and appeared like a recent development in the fields of FS and NS theory.

All these above literatures show that CSM is a hot topic in both practical and theoretical fields [8]-[19]. However, up to now, as far as we are aware, there is no research on the CSM and weighted CSM of IVBNHFS. Hence, in this paper, we focus on this issue and propose important CSM and weighted CSM of IVBNHFS.

\section{A. Knowledge Gap}

MADM approach appropriate to CSM and weighted CSM of IVBNHFS.

(e-mail: reneheli@yahoo.fr)

H. Z. Andriamanohisoa, University of Antananarivo, Madagascar. (e-mail: aheryzo@gmail.com).
Published on October 23, 2021.
H. E. Ramaroson, University of Antananarivo, Madagascar.

(corresponding e-mail: erichansise@gmail.com)

R. Rakotomanana, University of Antananarivo, Madagascar. 


\section{B. Research Issues}

i. Is it feasible to suggest a CSM for IVBNHFSs?

ii. Is it conceivable to engender a weighted CSM for IVBNHFSs?

iii. Is it achievable to develop a novel MADM approach based on the proposed CSM in IVBNHF setting?

iv. Is it realizable to set up a novel MADM strategy based on the proposed weighted CSM for IVBNHFSs in IVBNHF environment?

To do as such, the plan of this paper is coordinated as follows. The section 2 gives some knowledge preliminaries on IVBNHFS. The section 3 proposes CSM for IVBNHFS and their properties. And, the weighted CSM is investigated. In section 4, we introduce the novel similarity measures for MADM problem in IVBNHF environment. The section 5 suggests an illustrative case of MADM to demonstrate the effectiveness, workability, and appropriateness of the proposed MADM approach. The paper ends with some comparative study and concluding remarks in the section 6 and the section 7 , respectively.

\section{Mathematical Preliminaries}

\section{A. IVBNHFS [21]}

IVBNHFS is an effective tool to process the uncertain, inconsistent and hesitant information.

\section{Definition 1:}

Assume $X$ is a finite set which contains at least one element, an IVBNHFS $P$ on $X$ is described as:

$$
\left.P=\left\{\begin{array}{c}
x, t^{+}(x), i^{+}(x), f^{+}(x), \\
t^{-}(x), i^{-}(x), f^{-}(x)
\end{array}\right\rangle \mid x \in X\right\}
$$

\section{where:}

$t^{+}(x)=\left\{\gamma^{+} \mid \gamma^{+} \in t^{+}(x)\right\}$,

$i^{+}(x)=\left\{\delta^{+} \mid \delta^{+} \in i^{+}(x)\right\}$, and,

$f^{+}(x)=\left\{\eta^{+} \mid \eta^{+} \in f^{+}(x)\right\}$ are positive three membership functions expressed by a few closed intervals in the real unit interval $[0,1]$ which detail the truth or indeterminacy or falsity positive membership hesitant degree, and meet the following conditions:

$$
\begin{aligned}
& \gamma^{+}=\left[\gamma_{L}^{+}, \gamma_{U}^{+}\right] \in[0,1], \\
& \delta^{+}=\left[\delta_{L}^{+}, \delta_{U}^{+}\right] \in[0,1], \\
& \eta^{+}=\left[\eta_{L}^{+}, \eta_{U}^{+}\right] \in[0,1], \text { and: } \\
& \quad \quad 0 \leq \sup ^{m+}+\sup ^{m+}+\sup ^{m+} \leq 3
\end{aligned}
$$

$$
\begin{aligned}
& \text { where: } \\
& \gamma^{m+}=\bigcup_{\gamma^{+} \in t^{+}(x)} \max \gamma^{+}, \\
& \delta^{m+}=\bigcup_{\delta^{+} \in i^{+}(x)} \max \delta^{+}, \text {and } \\
& \eta^{m+}=\bigcup_{\eta^{+} \in f^{+}(x)} \max \eta^{+}
\end{aligned}
$$

And $t^{-}(x)=\left\{\gamma^{-} \mid \gamma^{-} \in t^{-}(x)\right\}$

$i^{-}(x)=\delta^{-} \mid \delta^{-} \in i^{-}(x)$, and,

$f^{-}(x)=\left\{\eta^{-} \mid \eta^{-} \in f^{-}(x)\right\}$ are negative three membership functions expressed by a few closed intervals in the real unit interval $[-1,0]$ which detail the truth or indeterminacy or falsity negative membership hesitant degree and meet the following conditions:

$$
\begin{aligned}
& \gamma^{-}=\left[\gamma_{L}^{-}, \gamma_{U}^{-}\right] \in[-1,0], \\
& \delta^{-}=\left[\delta_{L}^{-}, \delta_{U}^{-}\right] \in[-1,0], \\
& \eta^{-}=\left[\eta_{L}^{-}, \eta_{U}^{-}\right] \in[-1,0], \text { and: } \\
& \quad \quad-3 \leq \operatorname{supr}^{m-}+\sup ^{m-}+\sup ^{m-} \leq 0
\end{aligned}
$$

where

$\gamma^{m-}=U_{\gamma^{-} \in t^{-}(x)} \max \gamma^{-}$,

$\delta^{m-}=\bigcup_{\delta^{-} \in i^{-}(x)} \max \delta^{-}$, and,

$\eta^{m-}=\cup_{\eta^{-} \in f^{-}(x)} \max \eta^{-}$.

\section{B. IVBNHFS Properties}

1) Complement [21]

Definition 2:

Let

$$
A=\left\{\begin{array}{l}
{\left[t_{L A}^{+}, t_{U A}^{+}\right],\left[i_{L A}^{+}, i_{U A}^{+}\right],\left[f_{L A}^{+}, f_{U A}^{+}\right],} \\
{\left[t_{L A}^{-}, t_{U A}^{-}\right],\left[i_{L A}^{-}, i_{U A}^{-}\right],\left[f_{L A}^{-}, f_{U A}^{-}\right]}
\end{array}\right\}
$$

be an IVBNHFS, the complement $A^{C}$ of an IVBNHFS $A$ is:

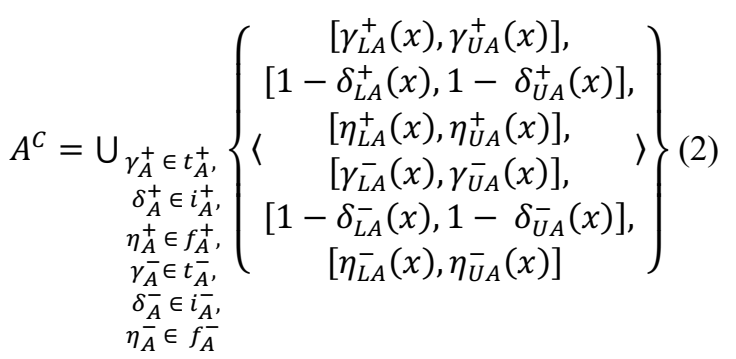

2) Intersection [21]

Definition 3:

Let

$A=\left\{\begin{array}{l}{\left[t_{L A}^{+}, t_{U A}^{+}\right],\left[i_{L A}^{+}, i_{U A}^{+}\right],\left[f_{L A}^{+}, f_{U A}^{+}\right],} \\ {\left[t_{L A}^{-}, t_{U A}^{-}\right],\left[i_{L A}^{-}, i_{U A}^{-}\right],\left[f_{L A}^{-}, f_{U A}^{-}\right]}\end{array}\right\}$, and,

$B=\left\{\begin{array}{l}{\left[t_{L B}^{+}, t_{U B}^{+}\right],\left[i_{L B}^{+}, i_{U B}^{+}\right],\left[f_{L B}^{+}, f_{U B}^{+}\right],} \\ {\left[t_{L B}^{-}, t_{U B}^{-}\right],\left[i_{L B}^{-}, i_{U B}^{-}\right],\left[f_{L B}^{-}, f_{U B}^{-}\right]}\end{array}\right\} ;$are two IVBNHFS, the intersection $A \cap B$ of a two IVBNHFS $A$ and $B$ is:

$$
\begin{gathered}
{\left[\wedge\left(\gamma_{L A}^{+}, \gamma_{L B}^{+}\right), \wedge\left(\gamma_{U A}^{+}, \gamma_{U B}^{+}\right)\right],} \\
{\left[\bigvee\left(\delta_{L A}^{+}, \delta_{L B}^{+}\right), \bigvee\left(\delta_{U A}^{+}, \delta_{U B}^{+}\right)\right],} \\
{\left[\bigvee\left(\eta_{L A}^{+}, \eta_{L B}^{+}\right), \bigvee\left(\eta_{U A}^{+}, \eta_{U B}^{+}\right)\right],} \\
{\left[\wedge\left(\gamma_{L A}^{-}, \gamma_{L B}^{-}\right), \wedge\left(\gamma_{U A}^{-}, \gamma_{U B}^{-}\right)\right],} \\
\gamma_{A}^{+} \in t_{A}^{+}, \delta_{A}^{+} \in i_{A}^{+}, \\
\eta_{A}^{+} \in f_{A}^{+}, \\
\gamma_{A}^{-} \in t_{A}^{-}, \\
\delta_{A}^{-} \in i_{A}^{-}, \\
\eta_{A}^{-} \in f_{A}^{-}, \\
\gamma_{B}^{+} \in t_{B^{\prime}}^{+}, \\
\delta_{B}^{+} \in i_{B}^{+}, \\
\eta_{B}^{+} \in f_{B^{\prime}}^{+}, \\
\gamma_{B}^{-} \in t_{B}^{-}, \\
\delta_{B}^{-} \in i_{B}^{-}, \\
\eta_{B}^{-} \in f_{B}^{-}
\end{gathered}
$$




\section{3) Union [21]}

Definition 4:

Let

$A=\left\{\begin{array}{l}{\left[t_{L A}^{+}, t_{U A}^{+}\right],\left[i_{L A}^{+}, i_{U A}^{+}\right],\left[f_{L A}^{+}, f_{U A}^{+}\right],} \\ {\left[t_{L A}^{-}, t_{U A}^{-}\right],\left[i_{L A}^{-}, i_{U A}^{-}\right],\left[f_{L A}^{-}, f_{U A}^{-}\right]}\end{array}\right\}$, and,

$B=\left\langle\begin{array}{c}{\left[t_{L B}^{+}, t_{U B}^{+}\right],\left[i_{L B}^{+}, i_{U B}^{+}\right],\left[f_{L B}^{+}, f_{U B}^{+}\right],} \\ {\left[t_{L B}^{-}, t_{U B}^{-}\right],\left[i_{L B}^{-}, i_{U B}^{-}\right],\left[f_{L B}^{-}, f_{U B}^{-}\right]}\end{array}\right\rangle ;$are two IVBNHFS,

the union $A \cup B$ of a two IVBNHFS $A$ and $B$ is:

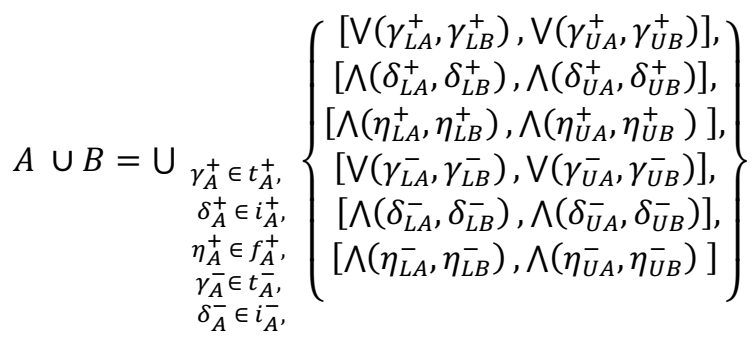

$$
\begin{aligned}
& \eta_{\bar{A}} \in f_{\bar{A}}^{-} \text {, } \\
& \gamma_{B}^{+} \in t_{B}^{+} \text {, } \\
& \delta_{B}^{+} \in i_{B}^{+} \text {, } \\
& \eta_{B}^{+} \in f_{B}^{+} \text {, } \\
& \gamma_{B}^{-} \in t_{B}^{-} \text {, } \\
& \delta_{B}^{-} \in i_{B}^{-} \text {, } \\
& \eta_{B}^{-} \in f_{B}^{-}
\end{aligned}
$$

\section{PROPOSED CSM FOR IVBNHFS}

For CS between two vectors, two FS, two IFS and for INS, see [8].

\section{A. CSM for IVBNHFS}

\section{Definition 5:}

Let $B_{1}$ and $B_{2}$, are two IVBNHFS in $\mathrm{X}=\left\{\mathrm{x}_{1}, \mathrm{x}_{2}, \ldots, \mathrm{x}_{\mathrm{n}}\right\}$.

$$
\begin{aligned}
& B_{1}=\left\{\begin{array}{c}
x_{i}, t_{1}^{+}\left(x_{i}\right), i_{1}^{+}\left(x_{i}\right) \\
\left\langle, f_{1}^{+}\left(x_{i}\right), t_{1}^{-}\left(x_{i}\right),\right\rangle \\
i_{1}^{-}\left(x_{i}\right), f_{1}^{-}\left(x_{i}\right)
\end{array} \mid x_{i} \in X, i=1,2, \ldots, n\right\} \\
& B_{2}=\left\{\begin{array}{c}
x_{i}, t_{2}^{+}\left(x_{i}\right), i_{2}^{+}\left(x_{i}\right), \\
\left\langle f_{2}^{+}\left(x_{i}\right), t_{2}^{-}\left(x_{i}\right),\right\rangle \\
i_{2}^{-}\left(x_{i}\right), f_{2}^{-}\left(x_{i}\right)
\end{array} \mid x_{i} \in X, i=1,2, \ldots, n\right\}
\end{aligned}
$$

$$
\begin{aligned}
& \gamma_{2 i \sigma(k)}^{+}=\left[\gamma_{L 2 i \sigma(k)}^{+}, \gamma_{U 2 i \sigma(k)}^{+}\right] \in t_{2}^{+}\left(x_{i}\right)\left(k=1,2, \ldots, l_{i}\right), \\
& \delta_{2 i \sigma(k)}^{+}=\left[\delta_{L 2 i \sigma(k)}^{+}, \delta_{U 2 i \sigma(k)}^{+}\right] \in i_{2}^{+}\left(x_{i}\right)\left(k=1,2, \ldots, p_{i}\right), \\
& \eta_{2 i \sigma(k)}^{+}=\left[\eta_{L 2 i \sigma(k)}^{+}, \eta_{U 2 i \sigma(k)}^{+}\right] \in f_{2}^{+}\left(x_{i}\right)\left(k=1,2, \ldots, q_{i}\right), \\
& \gamma_{2 i \sigma(k)}^{-}=\left[\gamma_{L 2 i \sigma(k)}^{-}, \gamma_{U 2 i \sigma(k)}^{-}\right] \in t_{2}^{-}\left(x_{i}\right)\left(k=1,2, \ldots, r_{i}\right), \\
& \delta_{2 i \sigma(k)}^{-}=\left[\delta_{L 2 i \sigma(k)}^{-}, \delta_{U 2 i \sigma(k)}^{-}\right] \in i_{2}^{-}\left(x_{i}\right)\left(k=1,2, \ldots, s_{i}\right), \\
& \eta_{2 i \sigma(k)}^{-}=\left[\eta_{L 2 i \sigma(k)}^{-}, \eta_{U 2 i \sigma(k)}^{-}\right] \in f_{2}^{-}\left(x_{i}\right)\left(k=1,2, \ldots, t_{i}\right), \\
& \text { where } l_{i}, p_{i}, q_{i}, r_{i}, s_{i}, t_{i},(\text { for } \mathrm{i}=2) \text { are the number of intervals } \\
& \text { in } t_{2}^{+}\left(x_{i}\right), i_{2}^{+}\left(x_{i}\right), f_{2}^{+}\left(x_{i}\right), t_{2}^{-}\left(x_{i}\right), i_{2}^{-}\left(x_{i}\right), \text { and } f_{2}^{-}\left(x_{i}\right) \\
& \text { respectively. }
\end{aligned}
$$

Established on the extension measure for FS, IFS and IVNFS, the CSM for IVBNHFS is introduced like that:$$
t_{1}^{+}(x)=\left\{\gamma_{1}^{+} \mid \gamma_{1}^{+} \in t_{1}^{+}(x)\right\},
$$$$
i_{1}^{+}(x)=\left\{\delta_{1}^{+} \mid \delta_{1}^{+} \in i_{1}^{+}(x)\right\},
$$$$
f_{1}^{+}(x)=\left\{\eta_{1}^{+} \mid \eta_{1}^{+} \in i_{1}^{+}(x)\right\},
$$$$
t_{2}^{+}(x)=\left\{\gamma_{2}^{+} \mid \gamma_{2}^{+} \in t_{2}^{+}(x)\right\} \text {, }
$$$$
i_{2}^{+}(x)=\left\{\delta_{2}^{+} \mid \delta_{2}^{+} \in i_{2}^{+}(x)\right\},
$$$$
f_{2}^{+}(x)=\left\{\eta_{2}^{+} \mid \eta_{2}^{+} \in i_{2}^{+}(x)\right\} \text {. }
$$

And

$t_{1}^{-}(x)=\left\{\gamma_{1}^{-} \mid \gamma_{1}^{-} \in t_{1}^{-}(x)\right\}$,

$i_{1}^{-}(x)=\left\{\delta_{1}^{-} \mid \delta_{1}^{-} \in i_{1}^{-}(x)\right\}$,

$f_{1}^{-}(x)=\left\{\eta_{1}^{-} \mid \eta_{1}^{-} \in i_{1}^{-}(x)\right\}$,

$t_{2}^{-}(x)=\left\{\gamma_{2}^{-} \mid \gamma_{2}^{-} \in t_{2}^{-}(x)\right\}$,

$i_{2}^{-}(x)=\left\{\delta_{2}^{-} \mid \delta_{2}^{-} \in i_{2}^{-}(x)\right\}$,

$f_{2}^{-}(x)=\left\{\eta_{2}^{-} \mid \eta_{2}^{-} \in i_{2}^{-}(x)\right\}$,

For $i=1,2, \ldots, n$ :

$$
\begin{aligned}
& \cos _{\mathrm{IVBNHFS}}\left(B_{1}, B_{2}\right)=\frac{\cos _{1}}{\cos _{2} \times \cos _{3}} \\
& \cos _{1}=\sum_{j=1}^{n}\left\{\begin{array}{c}
\frac{1}{l_{i}} \sum_{k=1}^{l_{i}}\left[\begin{array}{c}
\left(\gamma_{L 1 i \sigma(k)}^{+}+\gamma_{U 1 i \sigma(k)}^{+}\left(\gamma_{L 2 i \sigma(k)}^{+}+\gamma_{U 2 i \sigma(k)}^{+}\right)\right.
\end{array}\right] \\
+\frac{1}{p_{i}} \sum_{k=1}^{p_{i}}\left[\begin{array}{l}
\left(\delta_{L 1 i \sigma(k)}^{+}+\delta_{U 1 i \sigma(k)}^{+}\right) \\
\left(\delta_{L 2 i \sigma(k)}^{+}+\delta_{U 2 i \sigma(k)}^{+}\right)
\end{array}\right] \\
+\frac{1}{q_{i}} \sum_{k=1}^{q_{i}}\left[\begin{array}{l}
\left(\eta_{L 1 i \sigma(k)}^{+}+\eta_{U 1 i \sigma(k)}^{+}\right) \\
\left(\eta_{L 2 i \sigma(k)}^{+}+\eta_{U 2 i \sigma(k)}^{+}\right)
\end{array}\right] \\
+\frac{1}{r_{i}} \sum_{k=1}^{r_{i}}\left[\begin{array}{l}
\left(\gamma_{L 1 i \sigma(k)}^{-}+\gamma_{U 1 i \sigma(k)}^{-}\right) \\
\left(\gamma_{L 2 i \sigma(k)}^{-}+\gamma_{U 2 i \sigma(k)}^{-}\right)
\end{array}\right] \\
+\frac{1}{s_{i}} \sum_{k=1}^{s_{i}}\left[\begin{array}{c}
\left(\delta_{L 1 i \sigma(k)}^{-}+\delta_{U 1 i \sigma(k)}^{-}\right) \\
\left(\delta_{L 2 i \sigma(k)}^{-}+\delta_{U 2 i \sigma(k)}^{-}\right)
\end{array}\right] \\
+\frac{1}{t_{i}} \sum_{k=1}^{t_{i}}\left[\begin{array}{l}
\left(\eta_{L i i \sigma(k)}^{-}+\eta_{U 1 i \sigma(k)}^{-}\right) \\
\left(\eta_{L 2 i \sigma(k)}^{-}+\eta_{U 2 i \sigma(k)}^{-}\right)
\end{array}\right]
\end{array}\right\}, \\
& \cos _{2}=\sum_{j=1}^{n}\left\{\begin{array}{c}
\frac{1}{l_{i}} \sum_{k=1}^{l_{i}}\left[\left(\gamma_{L 1 i \sigma(k)}^{+}+\gamma_{U 1 i \sigma(k)}^{+}\right)^{2}\right] \\
+\frac{1}{p_{i}} \sum_{k=1}^{p_{i}}\left[\left(\delta_{L 1 i \sigma(k)}^{+}+\delta_{U 1 i \sigma(k)}^{+}\right)^{2}\right] \\
+\frac{1}{q_{i}} \sum_{k=1}^{q_{i}}\left[\left(\eta_{L 1 i \sigma(k)}^{+}+\eta_{U 1 i \sigma(k)}^{+}\right)^{2}\right] \\
+\frac{1}{r_{i}} \sum_{k=1}^{r_{i}}\left[\left(\gamma_{L 1 i \sigma(k)}^{-}+\gamma_{U 1 i \sigma(k)}^{-}\right)^{2}\right] \\
+\frac{1}{s_{i}} \sum_{k=1}^{s_{i}}\left[\left(\delta_{L 1 i \sigma(k)}^{-}+\delta_{U 1 i \sigma(k)}^{-}\right)^{2}\right] \\
+\frac{1}{t_{i}} \sum_{k=1}^{\frac{1}{2}}\left[\left(\eta_{L 1 i \sigma(k)}^{-}+\eta_{U 1 i \sigma(k)}^{-}\right)^{2}\right]
\end{array}\right\}
\end{aligned}
$$




$$
\cos _{3}=\sum_{j=1}^{n}\left\{\begin{array}{c}
\frac{1}{l_{i}} \sum_{k=1}^{l_{i}}\left[\left(\gamma_{L 2 i \sigma(k)}^{+}+\gamma_{U 2 i \sigma(k)}^{+}\right)^{2}\right] \\
+\frac{1}{p_{i}} \sum_{k=1}^{p_{i}}\left[\left(\delta_{L 2 i \sigma(k)}^{+}+\delta_{U 2 i \sigma(k)}^{+}\right)^{2}\right] \\
+\frac{1}{q_{i}} \sum_{k=1}^{q_{i}}\left[\left(\eta_{L 2 i \sigma(k)}^{+}+\eta_{U 2 i \sigma(k)}^{+}\right)^{2}\right] \\
+\frac{1}{r_{i}} \sum_{k=1}^{r_{i}}\left[\left(\gamma_{L 2 i \sigma(k)}^{-}+\gamma_{U 2 i \sigma(k)}^{-}\right)^{2}\right] \\
+\frac{1}{s_{i}} \sum_{k=1}^{s_{i}}\left[\left(\delta_{L 2 i \sigma(k)}^{-}+\delta_{U 2 i \sigma(k)}^{-}\right)^{2}\right] \\
+\frac{1}{t_{i}} \sum_{k=1}^{t_{i}}\left[\left(\eta_{L 2 i \sigma(k)}^{-}+\eta_{U 2 i \sigma(k)}^{-}\right)^{2}\right]
\end{array}\right\}
$$

Theorem 1:

The following equations are true:

(i) $0 \leq \cos _{\text {IVBNHFS }} \leq 1$

(ii) $\cos _{\text {IVBNHFS }}\left(B_{1}, B_{2}\right)=\cos _{2 H Z S}\left(B_{2}, B_{1}\right)$

(iii) $\cos _{\text {IVBNHFS }}\left(B_{1}, B_{2}\right)=1$ si $B_{1}=B_{2}$

Proof. The theorem is straightforward. $\square$

\section{B. Weighted CSM between IVBNHFS (WIVBNHFS)}

\section{Definition 6:}

Assume we recognize the weights of each element $x_{i}$, a weighted CSM between IVBNHFS $B_{1}$ and $B_{2}$ is suggested as follows:

$$
\cos _{\mathrm{WIVBNHFS}}\left(B_{1}, B_{2}\right)=\frac{\cos _{W 1}}{\cos _{W 2} \times \cos _{W 3}}
$$

where

$$
\cos _{w 1}=\sum_{j=1}^{n} w_{i}\left\{\begin{array}{c}
\frac{1}{l_{i}} \sum_{k=1}^{l_{i}}\left[\begin{array}{l}
\left(\gamma_{L 1 i \sigma(k)}^{+}+\gamma_{U 1 i \sigma(k)}^{+}\right) \\
\left(\gamma_{L 2 i \sigma(k)}^{+}+\gamma_{U 2 i \sigma(k)}^{+}\right)
\end{array}\right] \\
+\frac{1}{p_{i}} \sum_{k=1}^{p_{i}}\left[\begin{array}{l}
\left(\delta_{L 1 i \sigma(k)}^{+}+\delta_{U 1 i \sigma(k)}^{+}\right) \\
\left(\delta_{L 2 i \sigma(k)}^{+}+\delta_{U 2 i \sigma(k)}^{+}\right)
\end{array}\right] \\
q_{i} \sum_{k=1}^{q_{i}}\left[\begin{array}{l}
\left(\eta_{L 1 i \sigma(k)}^{+}+\eta_{U 1 i \sigma(k)}^{+}\right) \\
\left(\eta_{L 2 i \sigma(k)}^{+}+\eta_{U 2 i \sigma(k)}^{+}\right)
\end{array}\right] \\
+\frac{1}{r_{i}} \sum_{k=1}^{r_{i}}\left[\begin{array}{l}
\left(\gamma_{L 1 i \sigma(k)}^{-}+\gamma_{U 1 i \sigma(k)}^{-}\right) \\
\left(\gamma_{L 2 i \sigma(k)}^{-}+\gamma_{U 2 i \sigma(k)}^{-}\right)
\end{array}\right] \\
+\frac{1}{s_{i}} \sum_{k=1}^{s_{i}}\left[\begin{array}{l}
\left(\delta_{L 1 i \sigma(k)}^{-}+\delta_{U 1 i \sigma(k)}^{-}\right) \\
\left(\delta_{L 2 i \sigma(k)}^{-}+\delta_{U 2 i \sigma(k)}^{-}\right)
\end{array}\right] \\
+\frac{1}{t_{i}} \sum_{k=1}^{t_{i}}\left[\begin{array}{l}
\left(\eta_{L 1 i \sigma(k)}^{-}+\eta_{U 1 i \sigma(k)}^{-}\right) \\
\left(\eta_{L 2 i \sigma(k)}^{-}+\eta_{U 2 i \sigma(k)}^{-}\right)
\end{array}\right]
\end{array}\right\}
$$

$$
\cos _{w 2}=\sum_{j=1}^{n} w_{i}\left\{\begin{array}{c}
\frac{1}{l_{i}} \sum_{k=1}^{l_{i}}\left[\left(\gamma_{L 1 i \sigma(k)}^{+}+\gamma_{U 1 i \sigma(k)}^{+}\right)^{2}\right] \\
+\frac{1}{p_{i}} \sum_{k=1}^{p_{i}}\left[\left(\delta_{L 1 i \sigma(k)}^{+}+\delta_{U 1 i \sigma(k)}^{+}\right)^{2}\right] \\
+\frac{1}{q_{i}} \sum_{k=1}^{q_{i}}\left[\left(\eta_{L 1 i \sigma(k)}^{+}+\eta_{U 1 i \sigma(k)}^{+}\right)^{2}\right] \\
+\frac{1}{r_{i}} \sum_{k=1}^{r_{i}}\left[\left(\gamma_{L 1 i \sigma(k)}^{-}+\gamma_{U 1 i \sigma(k)}^{-}\right)^{2}\right] \\
+\frac{1}{s_{i}} \sum_{k=1}^{s_{i}}\left[\left(\delta_{L 1 i \sigma(k)}^{-}+\delta_{U 1 i \sigma(k)}^{-}\right)^{2}\right] \\
+\frac{1}{t_{i}} \sum_{k=1}^{\frac{1}{2}}\left[\left(\eta_{L 1 i \sigma(k)}^{-}+\eta_{U 1 i \sigma(k)}^{-}\right)^{2}\right]
\end{array}\right\}
$$

and

$$
\cos _{w 3}=\sum_{j=1}^{n} w_{i}\left\{\begin{array}{c}
\frac{1}{l_{i}} \sum_{k=1}^{l_{i}}\left[\left(\gamma_{L 2 i \sigma(k)}^{+}+\gamma_{U 2 i \sigma(k)}^{+}\right)^{2}\right] \\
+\frac{1}{p_{i}} \sum_{k=1}^{p_{i}}\left[\left(\delta_{L 2 i \sigma(k)}^{+}+\delta_{U 2 i \sigma(k)}^{+}\right)^{2}\right] \\
+\frac{1}{q_{i}} \sum_{k=1}^{q_{i}}\left[\left(\eta_{L 2 i \sigma(k)}^{+}+\eta_{U 2 i \sigma(k)}^{+}\right)^{2}\right] \\
+\frac{1}{r_{i}} \sum_{k=1}^{r_{i}}\left[\left(\gamma_{L 2 i \sigma(k)}^{-}+\gamma_{U 2 i \sigma(k)}^{-}\right)^{2}\right] \\
+\frac{1}{s_{i}} \sum_{k=1}^{s_{i}}\left[\left(\delta_{L 2 i \sigma(k)}^{-}+\delta_{U 2 i \sigma(k)}^{-}\right)^{2}\right] \\
+\frac{1}{t_{i}} \sum_{k=1}^{t_{i}}\left[\left(\eta_{L 2 i \sigma(k)}^{-}+\eta_{U 2 i \sigma(k)}^{-}\right)^{2}\right]
\end{array}\right\}
$$

with $w_{i} \in[0,1]$, for $i=1,2, \ldots, n$, and $\sum_{i=1}^{n} w_{i}=1$. If $w_{i}=\frac{1}{n}$, then, $C_{\text {WIVBNHFS }}\left(B_{1}, B_{2}\right)=C_{\text {WIVBNHFS }}\left(B_{1}, B_{2}\right)$.

Theorem 2:

The weighted CSM between two IVBNHFS $B_{1}$ and $B_{2}$ also has the following characteristics:

(i) $0 \leq \cos _{\text {WIVBNHFS }}\left(B_{1}, B_{2}\right) \leq 1$

(ii) $\cos _{\mathrm{WIVBNHFS}}\left(B_{1}, B_{2}\right)=\cos _{\mathrm{WIVBNHFS}}\left(B_{2}, B_{1}\right)$

(iii) $\cos _{\text {WIVBNHFS }}\left(B_{1}, B_{2}\right)=1$ if $B_{1}=B_{2}$

Proof. The theorem is straightforward. $\square$

\section{IVBNHF-MADM STRATEGIES BASED ON THE PROPOSED CSM}

This section presents the CSM for MADM problem in IVBNHF environment.

Consider $A=\left\{A_{1}, A_{2}, \ldots, A_{m}\right\}$, be a discrete set of $m$ achievable alternatives, and $C=\left\{C_{1}, C_{2}, \ldots, C_{n}\right\}$, be a set of criterions under consideration, and $w_{j}$ be the weight vector 
of the criterions such that $m$ and $n \geq 2, \quad 0 \leq w_{j} \leq 1$, and $\sum_{j=1}^{n} w_{j}=1$.

One novelty MADM strategy is presented in algorithmic form applying the following procedures.

\section{Proposed algorithm:}

Step 1: Present a Decision Matrix (DM)

The decision maker provides the IVBNHF-DM $D_{m x n}$, and assigns the rating of performance value of alternative $A_{i}$, with respect to the predefined criterions $C_{j}$ with reference to IVBNHF values, for $(i=1,2, \ldots, m ; j=1,2, \ldots, n)$ :

$$
p_{i j}=\left\{\begin{array}{l}
{\left[t_{L_{i j}}^{+}, t_{U_{i j}}^{+}\right],\left[i_{L_{i j}}^{+}, i_{U_{i j}}^{+}\right],\left[f_{L_{i j}}^{+} f_{U_{i j}}^{+}\right],} \\
{\left[t_{L_{i j}}^{-}, t_{U_{i j}}^{-}\right],\left[i_{L_{i j}}^{-}, i_{U_{i j}}^{-}\right],\left[f_{L_{i j}}^{-}, f_{U_{i j}}^{-}\right]}
\end{array}\right\}
$$

An IVBNHF-DM $D_{m x n}$ can be presented as follows:

$$
\begin{aligned}
& \begin{array}{lllll}
C_{1} & C_{2} & \ldots & C_{n}
\end{array} \\
& D_{m x n}=\begin{array}{c}
A_{1} \\
A_{2} \\
\vdots \\
\vdots \\
A_{m}
\end{array}\left[\begin{array}{cccc}
p_{11} & p_{12} & \ldots & p_{1 n} \\
p_{21} & p_{22} & \ldots & p_{2 n} \\
\vdots & \vdots & \ddots & \vdots \\
\vdots & \vdots & \vdots & \vdots \\
p_{m 1} & p_{m 2} & \ldots & p_{m n}
\end{array}\right]
\end{aligned}
$$

Step 2: Determine weights vector for criterions

Let decision maker predestine weights vector $w_{j}(j=$ $1,2, \ldots, n)$ of criterions $C_{j}(j=1,2, \ldots, n)$, for $w_{j} \in[0,1]$ and $\sum_{j=1}^{n} w_{j}=1$.

\section{Step 3: Propose an ideal alternative}

Let decision maker initiate to set up an ideal alternative of criterions values:

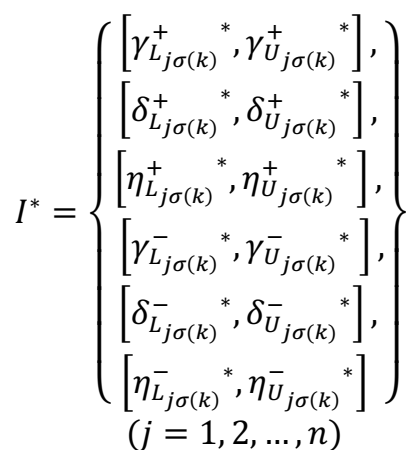

\section{Step 4: Compute CSM}

The weighted CSM $C_{\text {WIVBnhFs }}\left(A_{i}, I^{*}\right)$, between each alternative $A_{i}$, and the ideal solution $I^{*}$ are computed using (10), for $i=1,2, \ldots, m$ :

$$
\cos _{\mathrm{WIVBNHFS}}\left(A_{i}, I^{*}\right)=\frac{\cos _{W 1 *}}{\cos _{W 2 *} \times \cos _{W 3 *}}
$$

where

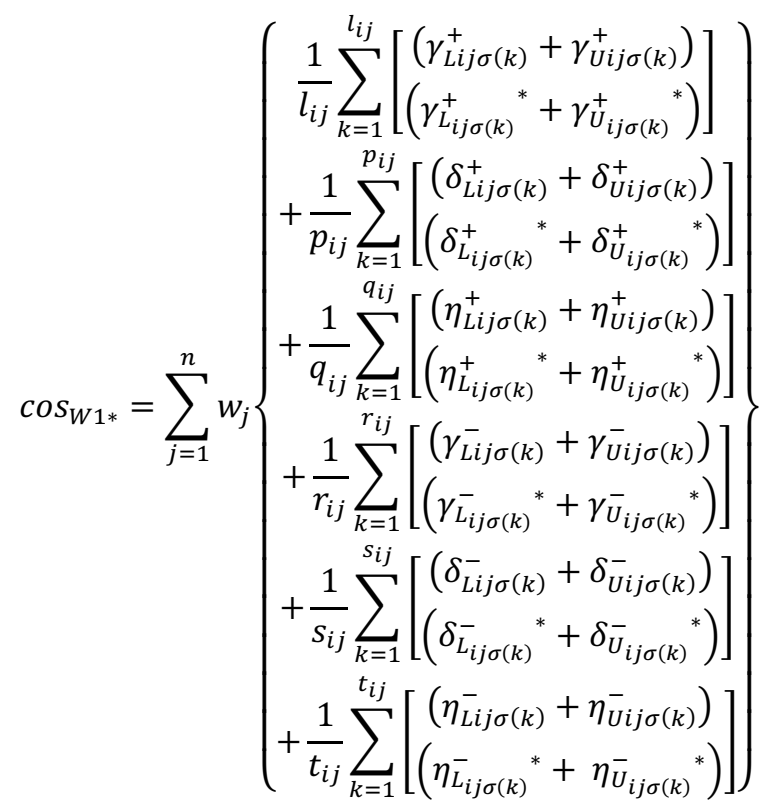

$$
\cos _{W 2 *}=\sum_{j=1}^{n} w_{j}\left\{\begin{array}{c}
\frac{1}{l_{i j}} \sum_{k=1}^{l_{i j}}\left[\left(\gamma_{L i j \sigma(k)}^{+}+\gamma_{U i j \sigma(k)}^{+}\right)^{2}\right] \\
+\frac{1}{p_{i j}} \sum_{k=1}^{p_{i j}}\left[\left(\delta_{L i j \sigma(k)}^{+}+\delta_{U i j \sigma(k)}^{+}\right)^{2}\right] \\
+\frac{1}{q_{i j}} \sum_{k=1}^{q_{i j}}\left[\left(\eta_{L i j \sigma(k)}^{+}+\eta_{U i j \sigma(k)}^{+}\right)^{2}\right] \\
+\frac{1}{r_{i j}} \sum_{k=1}^{r_{i j}}\left[\left(\gamma_{L i j \sigma(k)}^{-}+\gamma_{U i j \sigma(k)}^{-}\right)^{2}\right] \\
+\frac{1}{s_{i j}} \sum_{k=1}^{s_{i j}}\left[\left(\delta_{L i j \sigma(k)}^{-}+\delta_{U i j \sigma(k)}^{-}\right)^{2}\right] \\
+\frac{1}{t_{i j}} \sum_{k=1}^{\frac{1}{2}}\left[\left(\eta_{L i j \sigma(k)}^{-}+\eta_{U i j \sigma(k)}^{-}\right)^{2}\right]
\end{array}\right\}
$$

and

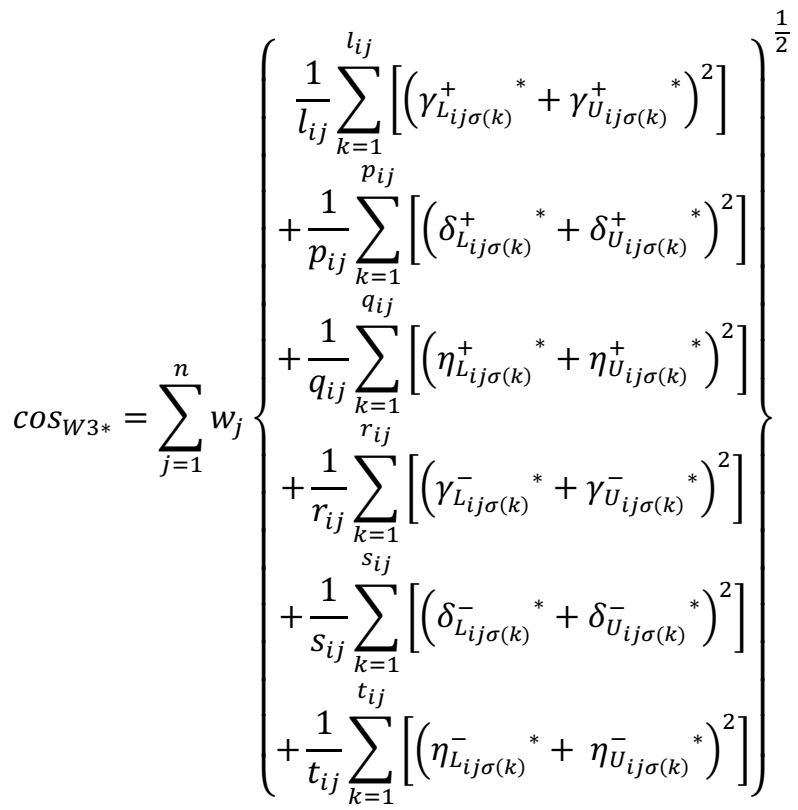




\section{Step 5: Rank all the alternative}

Rank all the alternative based on the decreasing order of $\cos _{\mathrm{WIVBNHFS}}\left(A_{i}, I^{*}\right)$, for $i=1,2, \ldots, m$.

Based on $\cos _{\text {WIVBNHFS }}\left(A_{i}, I^{*}\right)$, the highest value of $\cos _{\text {WIVBNHFS }}\left(A_{i}, I^{*}\right)$ indicates that $A_{i}$, for $i=1,2, \ldots, m$ is the best choice.

Step 6: Choose the best alternative

Choose the most desirable alternative in connection with the highest value of the $\cos _{\text {WIVBnhFs }}\left(A_{i}, I^{*}\right)$ in the step 5 .

The bigger value of $\cos _{\text {WIVBnhFs }}\left(A_{i}, I^{*}\right)$ reflects the preferable alternative.

Step 7: End

Stop.

\section{ILluSTRATIVE NumERICAL EXAMPLE}

The section $\mathrm{V}$ presents a numerical case study, adapted from reference [45]. Then, an IVBNHF-MADM problem is dispensed to validate the workability and effectiveness of the proposed CSM decision-making approach.

A company wants to start a business in one of the four manageable alternatives $\boldsymbol{A}_{\boldsymbol{i}}(\boldsymbol{i}=\mathbf{1}, \mathbf{2 , 3}, \mathbf{4}) . \boldsymbol{A}_{\mathbf{1}}, \boldsymbol{A}_{\mathbf{2}}, \boldsymbol{A}_{3}$, and $\boldsymbol{A}_{\mathbf{4}}$, represent four potential firms (car, food, computer and arms), respectively.

The four manageable alternatives need to be evaluated in agreement with the four criterions $C_{j}(j=1,2,3,4) . C_{1}$, $\boldsymbol{C}_{2}, \boldsymbol{C}_{3}$ and $\boldsymbol{C}_{\mathbf{4}}$ represent respectively the risk, the growth, the environmental impact, and the performance.

Assigned to the four criterions, the weight vector is $\boldsymbol{\omega}=$

$(0.24,0.26,0.26,0.24)^{T}$.

Now we use the MADM strategy based on the proposed CSM to get the most appropriate alternative.

Step 1: Acquire a DM

We can obtain the IVBNHF-DM $D_{4 x_{4}}$, as shown in Table I-IV.

The IVBNHF-DM $D_{4 \times 4}$ can be presented as follows:

TABLE I: IVBNHF DECISION MATRIX $\left(\boldsymbol{C}_{\boldsymbol{1}}\right)$

\begin{tabular}{|c|c|}
\hline & $C_{1}$ \\
\hline$A_{1}$ & $\begin{array}{c}\{\{[0.5,0.6]\} \\
\{[0.2,0.3],[0.3,0.4],[0.4,0.5]\} \\
\{[0.1,0.2],[0.2,0.3],[0.3,0.4] \\
[0.4,0.5],[0.5,0.6],[0.6,0.7]\} \\
\{[-0.2,-0.1]\} \\
\{[-0.6,-0.5],[-0.5,-0.4],[-0.4,-0.3],[-0.3,-0.2]\} \\
\{[-0.4,-0.3]\}\}\end{array}$ \\
\hline$A_{2}$ & $\begin{array}{c}\{\{[0.1,0.2]\}, \\
\{[0.3,0.4],[0.4,0.5],[0.5,0.6],[0.6,0.7],[0.7,0.8]\}, \\
\{[0.2,0.3],[0.3,0.4]\}, \\
\{[-0.5,-0.4],[-0.4,-0.3],[-0.3,-0.2]\}, \\
\{[-0.9,-0.8],[-0.8,-0.7],[-0.7,-0.6], \\
[-0.6,-0.5],[-0.5,-0.4],[-0.4,-0.3]\}, \\
\{[-0.6,-0.5],[-0.5,-0.4],[-0.4,-0.3], \\
[-0.3,-0.2],[-0.2,-0.1]\}\}\end{array}$ \\
\hline$A_{3}$ & $\begin{array}{c}\{\{[0.4,0.5],[0.5,0.6],[0.6,0.7],[0.7,0.8]\} \\
\{[0.4,0.5],[0.5,0.6]\} \\
\{[0.4,0.5],[0.5,0.6]\} \\
\{[-0.3,-0.2]\} \\
\{[-0.7,-0.6],[-0.6,-0.5]\} \\
\{[-0.5,-0.4]\}\}\end{array}$ \\
\hline$A_{4}$ & $\begin{array}{c}\{\{[0.6,0.7],[0.7,0.8],[0.8,0.9]\} \\
\{[0.3,0.4],[0.4,0.5],[0.5,0.6],[0.6,0.7],[0.7,0.8]\} \\
\{[0.5,0.6]\} \\
\{[-0.8,-0.7],[-0.7,-0.6],[-0.6,-0.5]\} \\
\{[-0.5,-0.4],[-0.4,-0.3],[-0.3,-0.2],[-0.2,-0.1]\} \\
\{[-0.2,-0.1]\}\}\end{array}$ \\
\hline
\end{tabular}

The TABLE I presents the first column of DM $D_{4 x 4}: p_{11}, p_{21}$, $p_{31}$ and $p_{41}$.

TABLE II: IVBNHF DECISION MATRIX $\left(\boldsymbol{C}_{2}\right)$

\begin{tabular}{|c|c|}
\hline & $C_{2}$ \\
\hline$A_{1}$ & $\begin{array}{c}\{[0.3,0.4],[0.4,0.5],[0.5,0.6],[0.6,0.7],[0.7,0.8], \\
[0.8,0.9]\},\{[0.1,0.2],[0.2,0.3],[0.3,0.4],[0.4,0.5], \\
\{[0.5,0.6],[0.6,0.7],[0.7,0.8]\}, \\
\{[0.2,0.3],[0.3,0.4],[0.4,0.5]\},\{[-0.8,-0.7]\}, \\
\{[-0.5,-0.4],[-0.4,-0.3],[-0.3,-0.2],[-0.2,-0.1]\} \\
\{[-0.4,-0.3],[-0.3,-0.2],[-0.2,-0.1]\}\}\end{array}$ \\
\hline$A_{2}$ & $\begin{array}{c}\{\{[0.2,0.3],[0.3,0.4],[0.4,0.5],[0.5,0.6],[0.6,0.7] \\
[0.7,0.8]\},\{[0.1,0.2],[0.2,0.3],[0.3,0.4]\} \\
\{[0.3,0.4]\} \\
\{[-0.5,-0.4],[-0.4,-0.3],[-0.3,-0.2],[-0.2,-0.1]\} \\
\{[-0.3,-0.2],[-0.2,-0.1]\} \\
\{[-0.9,-0.8],[-0.8,-0.7],[-0.7,-0.6],[-0.6,-0.5] \\
[-0.5,-0.4]\}\}\end{array}$ \\
\hline$A_{3}$ & $\begin{array}{c}\{\{[0.1,0.2],[0.2,0.3],[0.3,0.4],[0.4,0.5],[0.5,0.6]\}, \\
\{[0.3,0.4],[0.4,0.5],[0.5,0.6],[0.6,0.7],[0.7,0.8],[0.8,0.9]\}, \\
\{[0.3,0.4],[0.4,0.5]\}, \\
\{[-0.8,-0.7]\}, \\
\{[-0.4,-0.3]\}, \\
\{[-0.7,-0.6]\}\}\end{array}$ \\
\hline$A_{4}$ & $\begin{array}{c}\{\{[0.1,0.2]\}, \\
\{[0.8,0.9]\}, \\
\{[0.2,0.3],[0.3,0.4],[0.4,0.5],[0.5,0.6],[0.6,0.7]\}, \\
\{[-0.5,-0.4]\}, \\
\{[-0.6,-0.5],[-0.5,-0.4],[-0.4,-0.3]\}, \\
\{[-0.5,-0.4],[-0.4,-0.3]\}\}\end{array}$ \\
\hline
\end{tabular}

The TABLE II presents the second column of DM $D_{4 \times 4}$ : $p_{12}, p_{22}, p_{32}$ and $p_{42}$.

TABLE III: IVBNHF DECISION MATRIX $\left(\boldsymbol{C}_{\mathbf{3}}\right)$

\begin{tabular}{|c|c|}
\hline & $C_{3}$ \\
\hline$A_{1}$ & $\begin{array}{c}\{[0.1,0.2],[0.2,0.3],[0.3,0.4],[0.4,0.5],[0.5,0.6]\}, \\
\{[0.1,0.2],[0.2,0.3],[0.3,0.4],[0.4,0.5]\},\{[0.1,0.2], \\
[0.2,0.3],[0.3,0.4]\}, \\
\{[-0.5,-0.4],[-0.4,-0.3],[-0.3,-0.2]\} \\
\{[-0.7,-0.6],[-0.6,-0.5],[-0.5,-0.4], \\
\{[-0.4,-0.3]\} \\
\{[-0.4,-0.3],[-0.3,-0.2]\}\}\end{array}$ \\
\hline$A_{2}$ & $\begin{array}{c}\{\{[0.3,0.4]\} \\
\{[0.1,0.2],[0.2,0.3],[0.3,0.4],[0.4,0.5],[0.5,0.6]\} \\
\{[0.5,0.6],[0.6,0.7]\} \\
\{[-0.5,-0.4],[-0.4,-0.3],[-0.3,-0.2] \\
[-0.2,-0.1]\} \\
\{[-0.8,-0.7]\} \\
\{[-0.9,-0.8]\}\}\end{array}$ \\
\hline$A_{3}$ & $\begin{array}{c}\{\{[0.3,0.4],[0.4,0.5],[0.5,0.6],[0.6,0.7],[0.7,0.8], \\
[0.8,0.9]\}, \\
\{[0.2,0.3],[0.3,0.4],[0.4,0.5],[0.5,0.6],[0.6,0.7],[0.7,0.8]\}, \\
\{[0.2,0.3]\}, \\
\{[-0.5,-0.4]\}, \\
\{[-0.6,-0.5]\}, \\
\{[-0.7,-0.6]\}\end{array}$ \\
\hline$A_{4}$ & $\begin{array}{c}\{\{[0.2,0.3],[0.3,0.4],[0.4,0.5],[0.5,0.6],[0.6,0.7]\} \\
\{[0.5,0.6],[0.6,0.7],[0.7,0.8]\} \\
\{[0.8,0.9]\} \\
\{[-0.9,-0.8]\} \\
\{[-0.8,-0.7],[-0.7,-0.6],[-0.6,-0.5]\} \\
\{[-0.5,-0.4],[-0.4,-0.3],[-0.3,-0.2]\}\} \\
\end{array}$ \\
\hline
\end{tabular}

The TABLE III presents the third column of DM $D_{4 x 4}: p_{13}$, $p_{23}, p_{33}$ and $p_{43}$. 


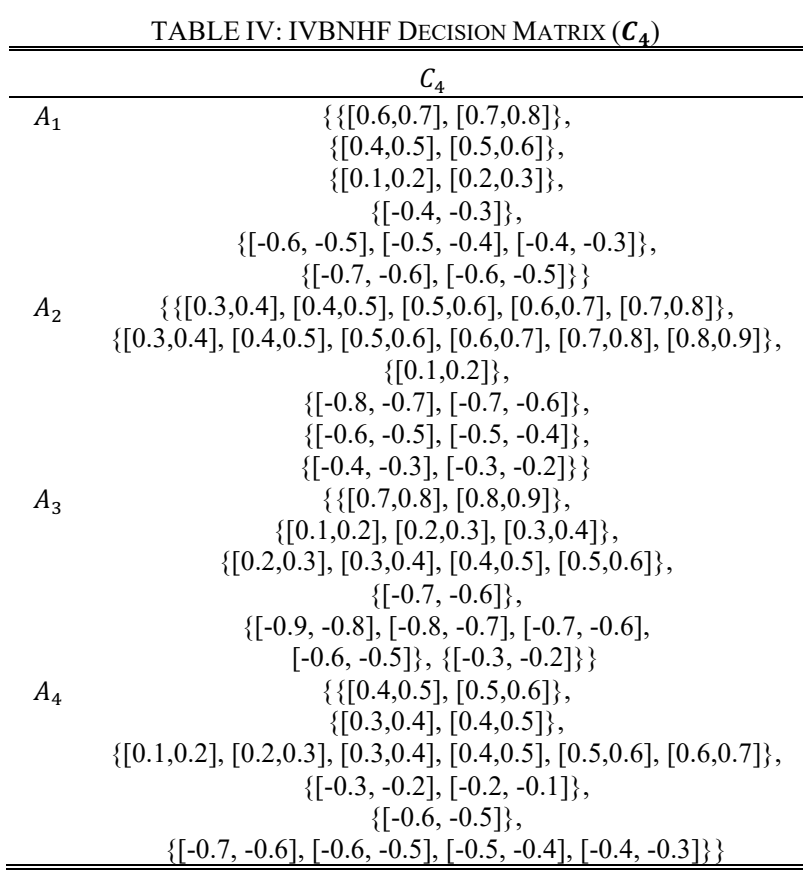

The Table IV presents the fourth column of DM $D_{4 \times 4}: p_{14}$, $p_{24}, p_{34}$ and $p_{44}$.

Step 2: Determine weights for criterions

The decision maker determined weights vector of criterions as $0.24,0.26,0.26,0.24$, respectively.

Step 3: Propose an ideal alternative

The ideal alternative $I^{*}$ is:

$$
\begin{gathered}
I^{*}=(\langle[1,1],[0,0],[0,0],[0,0],[-1,-1],[-1,-1]\rangle, \\
\langle[1,1],[0,0],[0,0],[0,0],[-1,-1],[-1,-1]\rangle, \\
\langle[1,1],[0,0],[0,0],[0,0],[-1,-1],[-1,-1]\rangle \\
\langle[1,1],[0,0],[0,0],[0,0],[-1,-1],[-1,-1]\rangle)
\end{gathered}
$$

\section{Step 4: Compute weighted CSM}

We refer to (10) for calculating $\cos _{\text {WIVBNhFs }}\left(A_{i}, I^{*}\right)$, (for $\mathrm{i}=1,2,3$, and 4).

We present the weighted CSM results in Table V.

TABLE V: COSINE SIMILARITY MEASURES RESULTS

\begin{tabular}{ccc}
\hline \hline Weighted CSM & $\begin{array}{c}\text { Measures } \\
\text { values }\end{array}$ & Ranking \\
\hline $\cos _{\text {WIVBNHFS }}\left(A_{1}, I^{*}\right)$ & 0.42 & 2 \\
$\cos _{\text {WIVBNHFS }}\left(A_{2}, I^{*}\right)$ & 0.39 & 3 \\
$\cos _{\text {WIVBNHFS }}\left(A_{3}, I^{*}\right)$ & 0.43 & 1 \\
$\cos _{\text {WIVBNHFS }}\left(A_{4}, I^{*}\right)$ & 0.32 & 4 \\
\hline \hline
\end{tabular}

Step 5: Rank all the alternative

Based on $\cos _{\text {WIVBNHFS }}\left(A_{i}, I^{*}\right)$ for $i=1,2,3$ and 4, we have $A_{3}>A_{1}>A_{2}>A_{4}$.

\section{Step 6: Choose the preferable alternative}

In connection with the highest value of the $\cos _{\text {WIVBNHFS }}\left(A_{i}, I^{*}\right)$ in the step 5 , the most preferable alternative is $A_{3}$.

\section{COMParative Study}

To further validate the feasibility of above this CSM MADM approaches, a comparative study was conducted with Deli 's methods in [45]. However, the results by utilizing different methods are shown in Table VI.
For the compared methods in [45], Deli and al. proposes two kinds of aggregation operators, the Interval Valued Bipolar Neutrosophic Weighted Average (IVBNWA) and Interval Valued Bipolar Neutrosophic Weighted Geometric (IVBNWG) operators [45].

\begin{tabular}{lccc}
\multicolumn{4}{c}{ TABLE VI: COMPARATIVE STUDY RESULTS } \\
\hline \hline Methods & Final Ranking & $\begin{array}{c}\text { Preferable } \\
\text { Alternative }\end{array}$ & $\begin{array}{c}\text { Worst } \\
\text { Option }\end{array}$ \\
\hline \hline IBNS [45] & $A_{2}>A_{3}>A_{1}>A_{4}$ & $A_{2}$ & $A_{4}$ \\
CSM of & $A_{3}>A_{1}>A_{2}>A_{4}$ & $A_{3}$ & \\
IVBNHFS &
\end{tabular}

For different methods: Deli 's methods in [45], IVBNHFWA or IVBNHFWG, as we can see from Table VI, the final ranking may be different each other. Thus, according to the results obtained by utilizing different methods, if the IVBNS operators [45] are used, the desirable choice is $A_{2}$, and if our method is utilized, the best alternative is $A_{3}$. Then, our CSM order of preference for the four manageable alternatives is in disagreement with the Deli 's method [45] result. On the other hand, the worst option is always $A_{4}$. So, we really need another similarity measure to confirm the results.

\section{CONCLUSION}

The paper presented the concept of CSM for MADM of IVBNHFS. The proposed CSM, which is the first method expanded to find out the preferable alternative under IVBNHF setting, is utilized to promote a new MADM models. However, we presented CSM under IVBNHF environment and demonstrated some of their basic characteristics. Furthermore, the weighted CSM was applied to a MADM approach in which the attributes values take the form of Interval Valued Bipolar Neutrosophic Hesitant Fuzzy Elements (IVBNHFEs) with respect to the alternatives and the criteria weights are known information. We utilize a new algorithm to prioritize the alternatives and determine the preferable one. Finally, an illustrative numerical adapted from [45] is given to indicate the applicability and productiveness of the proposed MADM strategy. Therefore, the proposed MADM strategy under IVBNHF setting is more appropriate for real scientific and engineering cases. In the future, we'll introduce a novel Analytic Hierarchy Process (AHP) approach or a new conjoint analysis (CA) with IVBNHFS. Also, based on CSM, we'll propose an Interval Valued Bipolar Neutrosophic Hesitant Fuzzy - Analytic Hierarchy Process (IVBNHFS-AHP) and an Interval Valued Bipolar Neutrosophic Hesitant Fuzzy - Conjoint Analysis (IVBNHFS-CA). In further work, we'll elaborate some more similarity measures and practiced them to MADM case, fault diagnosis, medical diagnosis, pattern recognition or other areas.

\section{ACKNOWLEDGMENT}

The authors would like to stretch out their appreciation to the editor of the European Journal of Mathematics and Statistics, and to the reviewers for their important advice. 


\section{REFERENCES}

[1] L. A. Zadeh, "Fuzzy sets", Information and Control 8: 338-353, 1965.

[2] K. T. Atanassov, "Intuitionistic fuzzy sets." Fuzzy Sets and Systems 20: 87-96, 1986.

[3] F. Smaramdache, "A unifying field in logics. Neutrosophy", Neutrosophic probability set and logic. Rehoboth: American research press 1998.

[4] W. R. Zhang, "Bipolar fuzzy sets", In Proceedings of the 1998 IEEE International Conference on Fuzzy Systems, Anchorage, AK, USA, 4 9; 835-840, 1998.

[5] V. Torra, "Hesitant fuzzy sets". Int J Intell Syst 25:529-539, 2010.

[6] J. Ye, "Multiple-attribute decision-making method under a singlevalued neutrosophic hesitant fuzzy environment", J Intell. Syst 24(1): 23-36, 2015.

[7] R. M. Hashim, M. Gulistan, and F. Smarandache, "Applications of neutrosophic bipolar fuzzy sets in hope foundation for planning to build a children hospital with different types of similarity measures", Symmetry, 10, 331, 2018.

[8] S. Broumi, F. Smarandache, "Cosine Similarity Measure of Interval Valued Neutrosophic Sets", Neutrosophic Sets and Systems, 5, 15-20, 2014.

[9] P. Biswas, S. Pramanik, and B. C. Giri, "Cosine Similarity Measure Based Multi-Attribute Decision-making with Trapezoidal Fuzzy Neutrosophic Numbers", Neutrosophic Sets and Systems 8, 47-57, 2014.

[10] S. Pramanik, K. Mondal, "Cosine Similarity Measure of Rough Neutrosophic Sets and Its Application In Medical Diagnosis", Global Journal of Advanced Research 2(1): 212-220, 2015.

[11] Z. Lu and J. Ye, "Cosine Measures of Neutrosophic Cubic Sets for Multiple Attribute Decision-Making”, Symmetry 9, 121, 2017.

[12] E. Bolturk, C. Kahraman, "A novel interval-valued neutrosophic AHP with cosine similarity measure", Soft Computing, part of Springer Nature, 22-4941-58, 2018.

[13] D. Liu, X. Chen and D. Peng, "Cosine distance measure between neutrosophic hesitant fuzzy linguistic sets and its application in multiple criteria decision making", Symmetry 10, 602, 2018.

[14] H. Garg, "An improved cosine similarity measure for intuitionistic fuzzy sets and their applications to decision-making process", Hacettepe Journal of Mathematics and Statistics 47(6): 1578-1594, 2018.

[15] D. Liu, X. Chen, and D. Peng, "Cosine similarity measure between hybrid intuitionistic fuzzy sets and its application in medical diagnosis", Hindawi, Computational and Mathematical Methods in Medicine 2018, 7 pages, 2018.

[16] A. E. Samuel, R. Narmadhagnanam, "Cosine exponential distance of single valued neutrosophic multi-sets in medical diagnosis", Journal of Engineering Research and Application 8(6) (Part -I), 26-29, 2018.

[17] A. E. Samuel, R. Narmadhagnanam, "Cosine logarithmic distance of single valued neutrosophic sets in medical diagnosis", International Journal of Engineering, Science and Mathematics, 7(6), 2018.

[18] C. Fan, E. Fan, and J. Ye, "Cosine measure of single-valued neutrosophic multisets for multiple attribute decision-making”, Symmetry, 10, 154, 2018.

[19] M. Abdel-Basset, M. Mohamed, M. Elhoseny, L. H.Son, F. Chiclana, H. Zaieda, "Cosine similarity measures of bipolar neutrosophic set for diagnosis of bipolar disorder diseases", Artificial Intelligence in Medicine 101, 101735.

[20] A. Al-Quran, A. Awang, M. Ali, and L. Abdullah, "Hesitant bipolarvalued neutrosophic set: formulation, theory and application," doi: 10.1109/ACCESS.2019.2946985, IEEE Access, 7, 176099-114, 2019.

[21] H. E. Ramaroson, H. Z. Andriamanohisoa, "Some operations and properties of interval valued bipolar neutrosophic hesitant fuzzy set (IVBNHFS)", International Journal of Advance Research and Innovative Ideas in Education, 28(2): 272-89, 2021.

[22] R. M. Hashim, M. Gulistan, I. Rehman, N. Hassan, A.M. Nasruddin, "Neutrosophic bipolar fuzzy set and its application in medicines preparations", Neutrosophic Sets and Systems, University of New Mexico 31, 86-100, 2020.

[23] N. A. Nabeeh, A. Abdel-Monem, A. Abdelmouty, "A novel methodology for assessment of hospital service according to BWM, MABAC, PROMETHEE II", Neutrosophic Sets and Systems, University of New Mexico 31, 2020.

[24] M. A. Malik, H. Rashmanlou, M. Shoaib1, R. A. Borzooei, M. Taheri, S. Broumi, "Study on bipolar single-valued neutrosophic graphs with novel application", Neutrosophic Sets and Systems 32, 221-268, 2020.A. Mukherjee, R. Das, "Neutrosophic bipolar vague soft set and its application to decision making problems", Neutrosophic Sets and Systems 32, 410-424, 2020.

[25] M. Mohanasundari, K. Mohana, "Quadripartitioned single valued neutrosophic Dombi weighted aggregation operators for multiple attribute decision making", Neutrosophic Sets and Systems 32, 107$122,2020$.

[26] Riaz, M., Naeem, K., Zareef, I., and Afzal, D., "Neutrosophic N-soft sets with TOPSIS method for multiple attribute decision making", Neutrosophic Sets and Systems 32, 146-170, 2020.

[27] H. Kamaci, "Neutrosophic cubic Hamacher aggregation operators and their applications in decision making", Neutrosophic Sets and Systems 33, 234-255, 2020

[28] A. Salama, A. Sharaf Al-Din, I. Abu Al-Qasim, R. Alhabib, and M. Badran, "Introduction to decision making for neutrosophic environment, Study on the Suez Canal Port, Egypt", Neutrosophic Sets and Systems 35, 22-44, 2020.

[29] M. Muthumeenakshi, S. Jafari, and P. Muralikrishna, "An ideal decision making on neutrosophic Qfuzzy setting", Neutrosophic Sets and Systems 36, 319-327, 2020.

[30] S. D. Álvarez-Gómez, J. F. Goyes-García, and B. Pinda-Guanolema, "Linking neutrosophic ahp and neutrosophic social choice theory for group decision making", Neutrosophic Sets and Systems 37, 385-394, 2020.

[31] R. M. Zulqarnain, X. L. Xin, M. Saeed, F. Smarandache, and N. Ahmad, "Generalized neutrosophic TOPSIS to solve multi-criteria decision-making problems", Neutrosophic Sets and Systems 38, 276292, 2020.

[32] D. Ajay, J. Aldring, S. Nivetha, "Neutrosophic cubic fuzzy Dombi hamy mean operators with application to multi-criteria decision making", Neutrosophic Sets and Systems 38, 293-316, 2020.

[33] M. Saeed, M. Saqlain, and A. Mehmood, "Application of similarity measure on m-polar interval-valued neutrosophic set in decision making in sports", Neutrosophic Sets and Systems 38, 317-334, 2020.

[34] M. Saqlain, A. Hamza, M. Saeed, and R. M. Zulqarnain, "Aggregate, arithmetic and geometric operators of octagonal neutrosophic numbers and its application in multi-criteria decision-making problems", Springer Book Series 2020.

[35] R. M. Zulqarnain, X. L. Xin, B. Ali, S. Broumi, S. Abdal and M. I. Ahamad, "Decision-making approach based on correlation coefficient with its properties under interval-valued neutrosophic hypersoft set environment", Neutrosophic Sets and Systems 40, 12-28, 2021.

[36] S. Şahin, A. Kargın and M. Yücel, "Hausdorff measures on generalized set valued neutrosophic quadruple numbers and decision-making applications for adequacy of online education", Neutrosophic Sets and Systems 40, 86-116, 2021.

[37] F. Al-Sharqi, A. Al-Quran, A. G. Ahmad and S. Broumi, "Intervalvalued complex neutrosophic soft set and its applications in decisionmaking", Neutrosophic Sets and Systems 40, 149-168, 2021.

[38] R. M. Zulqarnain, X. L. Xin, M. Saqlain, F. Smarandache and M. I. Ahamad, "An integrated model of neutrosophic topsis with application in multicriteria decision-making problem", Neutrosophic Sets and Systems 40, 253-269, 2021.

[39] A. U. Rahman, M. Saeed and A. Dhital, "Decision making application based on neutrosophic parameterized hypersoft set theory", Neutrosophic Sets and Systems 41, 1-14, 2021.

[40] V. Chinnadurai, M. P. Sindhu and K. Bharathivelan, "An introduction to neutro-prime topology and decision-making problem", Neutrosophic Sets and Systems 41, 146-167, 2021.

[41] A. M. El-hosiny, H. El-Ghareeb, B. T. Shabana and A. AbouElfetouh, "Sustainable energy production's spatial determination framework, based on multi criteria decision making and geographic information system under neutrosophic environment: a case study in Egypt", Neutrosophic Sets and Systems 42, 136-156, 2021.

[42] M. Ihsan, A. U. Rahman, M. Saeed, "Hypersoft expert set with application in decision making for recruitment process", Neutrosophic Sets and Systems 42, 191-207, 2021.

[43] M. Abdel-Basset, M. Mohamed, "Multi-criteria group decision making based on neutrosophic analytic hierarchy process: suggested modifications", Neutrosophic Sets and Systems 43, 247-254, 2021.

[44] I. Deli, Y. Şubaş, F. Smarandache, and M. Ali, "Interval valued bipolar neutrosophic sets and their application in decision making". Avalaible on https://www.researchgate.net/publication/289587637, 2016. 